\title{
Progressive Multifocal Leukoencephalopathy (PML) - a Case Report.
}

Khan BS1, Prof. Brig. (Retd) Alam J2

\begin{abstract}
Progressive Multifocal Leukoencephalopathy (PML) is a rare and usually fatal viral disease occurring almost exclusively in people with severe immune deficiency. This is a demyelinating disease of the central nervous system (CNS) that usually leads to death or severe disability. Patients usually present with focal CNS abnormalities like; hemiparesis, apathy, and confusion. Multifocal white matter lesions without mass effect lead to the diagnosis of PML. In this article we present a review of literature and report of a case of PML in which the patient's clinical status and MR findings are typical.
\end{abstract}

\section{Introduction}

\section{Case report}

A young man of 37 yrs who is HIV positive, presented with inability to walk and talk for 3 days. He had headache for 20 days and fever for one month.

On physical examination the patient's GCS was $8 / 15$, both pupils $2 \mathrm{~mm}$ and reacting. Bilateral planter reduced. BP was 130/90 mmHg. Crepitations were noted bilaterally in the chest. Neck was fairly rigid.

Blood profile was unremarkable except slightly high TLC. Anti HIV I/II rapid test were positive. AFB stain (Z.N) was negative and no malarial parasite was found. Toxoplasma antibody (IgG \& IgM) ELISA method was negative.

MRI of brain with contrast was performed in our hospital (using 1.5 Tesla Siemens MRI machine) MRI of brain showed multiple non enhancing white matter lesions in the right thalamo-ganglionic, left capsule-ganglionic region (Fig 2), body of corpus callosum (Fig 1) and in both cerebral hemispheres. No mass effect was noted. Patient was diagnosed as PML radiologically.

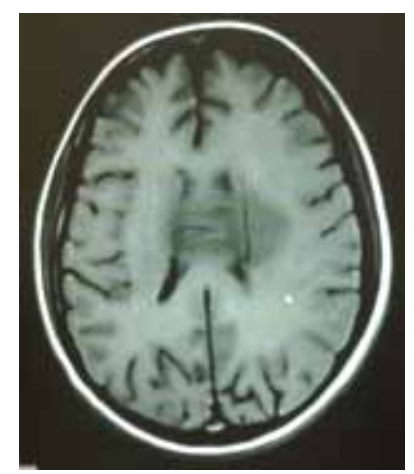

Fig 1: T1 Wt image shows low signal intensity areas in left corona radiata and body of corpus callosum.

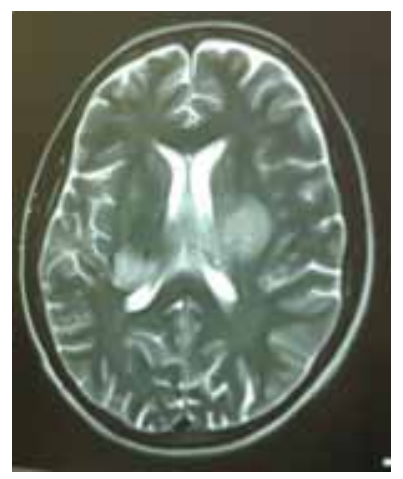

Fig 2: T2 Wt image shows high signal intensity areas in right thalamo-ganglionic and left capsule-ganglionic region. 


\section{Discussion}

Progressive Multifocal Leukoencephalopathy (PML) also known as progressive multifocal leucoencephalitis, is a rare and usually fatal viral disease that is characterized by progressive damage (-pathy) or inflammation of the white matter (- leuko-) of the brain (-encepahlo-) at the multiple locations (multifocal). An estimated 2-7\% of people with HIV disease will develop PML.

The cause of PML is a type of polyomavirus called the JC virus (JCV), after the initials of the patient from whose tissue the virus was first successfully cultured. Recent publications indicate $39 \%{ }^{1}$ to $58 \%{ }^{2}$ of the general population are seropositive for antibodies to JCV, indicating current or previous infection with virus. The virus can cause persistent asymptomatic infection in approximately one third of the adult population, based on viral shedding into the urine from the site of asymptomatic infection in the kidney. The virus causes disease only when the immune system has been severely weakened.

It occurs almost exclusively in people with severe immune deficiency, such as transplant patients on immunosuppressive medications, patients receiving certain kinds of chemotherapy or AIDS.

PML is caused by the reactivation of the JC virus, a polyomavirus that resides in latent form in $70-90 \%$ of the adult population worldwide. JC virus usually remains latent, typically only causing PML in immunocompromised patients. PML is a demyelinating disease, in which the myelin sheath covering the axons of nerve cells is gradually destroyed, impairing the transmission of nerve impulses. It affects the white matter, which is mostly composed of axons from the outermost parts of the brain (cortex). PML destroys oligodendrocytes and produces intranuclear inclusions.
PML is similar to another demyelinating disease, multiple sclerosis, but progresses much more quickly. The median survival of patients with PML is 6 months and in 9\% of patients, survival exceeds 1 year. 6

About 45\% of people with PML experience vision problems, most often a blindness affecting half of the visual field of each eye. Mental impairment affects about 38\% of people with PML. Eventually, about 75\% experience extreme weakness and other symptoms include lack of coordination, paralysis on one side of the body (hemiparesis) and problems in speaking or using language as seen in our case.

MRI offers superior sensitivity in the detection and characterization of the lesions though the diagnosis may be suspected by CT scan. On T2 W images the lesions appear hyperintense and typically involve the paraventricular and subcortical white matter having scalloped lateral margin when they involve the subcortical white matter predominantly in the parietooccipital lobes. Lesions are more conspicuously visualised in the FLAIR sequences, appearing hyperintense against a background of suppressed CSF signal intensity. Mass effect is infrequently described and usually minimal and correlation with shorter survival when seen on initial studies. The pattern may be unilateral, but more often bilateral and asymmetrical with involvement of posterior fossa as seen in our case. PML may also involve the basal ganglia and deep white nuclei as there are white matter fibers in these regions. The lesions typically do not enhance and do not have mass effect; however, there have been some reports of peripheral mild or diffuse enhancement. In those cases 
enhancement suggest good immune response and hence improve prognosis. There have been reports with lesions having cystic component suggesting tumors or abscess. ${ }^{3}$ In MR Spectroscopy findings are not specific. However there have been reports which showed broad decrease in CBF by SPECT in PML patients. ${ }^{4}$ On MR Spectroscopy there is reduced N-Acetylaspartate (NAA) with significant decreased NAA / Creathine ratio with increase choline and increased lactate peak. ${ }^{5}$ Nuclear imaging and angiography has no role in the diagnosis of the disease.

The CSF biochemistry and cytology is normal. In CSF, detection of JC virus by PCR is diagnostic. The sensitivity is $70 \%$ and specificity is 90-100\%. In negative PCR for JC virus repeat CSF examination or brain biopsy is advocated which is confirmatory diagnosis. ${ }^{5}$

\section{Reference}

1. Jaime M Kean, Suchitra Rao, Michael Wang, and Robert L Garcea. Seroepidemiology of human polyomaviruses. 2009. PLoS Pathog. 5(3):e1000363.
2. Adrian Egli, Laura Infanti, Alexis Dumoulin, Andreas Buser, Jacqueline Samaridis, Christine Stebler et al. Prevalence of poly omavirus BK and JC infection and replication in 400 healthy blood donors. The Journal of Infectious Diseases. 2009;199:837- 846.

3. Newton HB, Makley M, Slivka AP, Li J. Progressive multifocal leukoencephalopathy presenting as multiple enhancing lesions on MRI: case report and literature review. J Neuro Imaging. 1995 April;5(2):125-8.

4. Takase K, Ohyagi Y, Furuya H, Nagashima K, Taniwaki T, Kira J. A case of progressive multifocal leukoencephalopathy presenting white matter MRI lesions extending over the cerebral cortex and a marked decrease in cerebral blood flow on SPECT, and associated with HTLV-I infection. Rinsho Shinkeigaku. 2005 Jun; 45(6):426-30.

5. S Raina, S S Kaushal, D Gupta, P Himral, N Sawal+, V Sood + et al. Progressing Multifocal Leukoencephalopathy - As a presenting manifestation of AIDS. JAPI. 2007 Nov; 55.

6. Berger JR, Pall L, Lanska D, Whiteman M. Progressive leukoencephalopathy in patients with HIV infection. J Neurovirol. 1998 Feb;4(1):59-68. 